\title{
ESTUDO IN SILICO DO POTENCIAL DE FÁRMACOS AZÓLICOS SOBRE SARS-COV-2: UMA ABORDAGEM QUÍMICA-MEDICINAL
}

\author{
IN SILICO STUDY OF THE POTENTIAL OF AZOLIC DRUGS ON SARS-COV-2: A MEDICINAL \\ CHEMISTRY APPROACH
}

DOI: http://dx.doi.org/10.16891/2317-434X.v8.e3.a2020.pp636-648

Recebido em: 31.07 .2020 | Aceito em: 06.09.2020

Welson Vicente da Silva*a, Wesley Vicente da Silvab, Vanderlan Nogueira Holandac

Centro Universitário Maurício de Nassau - UNINASSAUa Centro Universitário UniFBV Wyden Universidade Federal de Pernambuco - UFPEc *E-mail: welson1535@hotmail.com

\section{RESUMO}

A doença do Novo Coronavírus 2019 (COVID-19) é causada pelo agente etiológico SARS-CoV-2 e até o momento, não existem medicamentos antivirais com eficácia comprovada contra o vírus. Neste cenário, a Organização Mundial de Saúde adotou estratégias de reposicionamentos de medicamentos para a busca de um tratamento contra a COVID-19. Dessa forma, medicamentos antifúngicos contendo grupos derivados de azóis chamam atenção da comunidade científica pelo seu potencial farmacológico já estabelecido. Fármacos como fluconazol, voriconazol e itraconazol apresentam um grupo triazol e o cetoconazol um grupamento imidazol em suas estruras químicas, comumente relacionados ao efeito biológico. A análise in silico é o primeiro passo para investigar a possibilidade de reposicionamento de fármacos já utilizados na prática clínica, além disso essa técnica permite a triagem de moléculas a fim de buscar o melhores compostos e com potencial terapêutico. Dessa forma, o presente trabalho teve por objetivo analisar o perfil farmacocinético, físico-químico e toxicológico in silico, bem como o estudo da interação destes compostos sobre alvos proteicos do SARS-CoV-2. As análises mostraram que somente os compostos cetoconazol e itraconazol violaram a regra de Lipinski. A análise de ancoragem molecular revelou que o fluconazol e voriconazol apresentaram valores favoráveis de energia livre para a interação com a protease principal do SARSCoV-2 e proteína do nucleocapsídeo. Em conjunto, esses resultados apontam para o potencial de estruturas formasdas por grupos triazólicos como candidatos a terapia contra o novo coronavírus.

Palavras-chave: Reposicionamento de fármacos; Fluconazol; Vorizonazol.

\section{ABSTRACT}

New Coronavirus 2019 (COVID-19) disease is caused by the etiologic agent SARS-CoV-2 and so far, there are no antiviral drugs with proven effectiveness against the virus. In this scenario, the World Health Organization has adopted strategies for repositioning medications to seek treatment against COVID-19. Thus, antifungal drugs containing groups derived from azoles draw the attention of the scientific community for their already established pharmacological potential. Drugs such as fluconazole, voriconazole and itraconazole have a triazole group and ketoconazole an imidazole group in their chemical structures, commonly related to the biological effect. In silico analysis is the first step to investigate the possibility of repositioning drugs already used in clinical practice, in addition this technique allows the screening of molecules in order to search for the best compounds and with therapeutic potential. Thus, the present study aimed to analyze the pharmacokinetic, physical-chemical and toxicological profile in silico, as well as the study of the interaction of these compounds on SARS-CoV-2 protein targets. The analyzes showed that only the compounds ketoconazole and itraconazole violated Lipinski's rule. Molecular anchorage analysis revealed that fluconazole and voriconazole showed favorable values of free energy for interaction with the main SARS-CoV-2 protease and nucleocapsid protein. Together, these results point to the potential of structures formed by triazole groups as candidates for therapy against the new coronavirus.

Keyword: Repositioning of drugs; Fluconazole; Voriconazole. 


\section{INTRODUÇÃO}

A doença causada pelo novo coronavírus 2019 (COVID-19) é uma infecção provocada pelo vírus da Síndrome Respiratória Aguda Grave do Coronavírus 2 (SARS-CoV-2), recentemente identificado e caracterizado após um surto no final de dezembro de 2019, em Huhan, China (Wang et al., 2020). Devido ao alto potencial de transmissibilidade, essa doença se espalhou rapidamente por diversos países, resultando na classificação oficial como pandemia pela Organização Mundial de Saúde (OMS) (Ransing et al., 2020). Além disso, até 24 de julho de 2020 o número de infecções confirmadas ultrapassa 15 milhões, com mais de 628 mil mortes no mundo. $\mathrm{O}$ alto número de indivíduos contaminados com o vírus indica que algumas pessoas podem não apresentar sintomas da doença e mesmo assim transmitirem o vírus (Furukawa et al., 2020). Os sintomas associados podem variar de leve a grave, dos quais destacam-se: febre, tosse, mialgia ou fadiga, pneumonia e aumento da dispneia. Dentre os sintomas menos comuns estão: dor de cabeça, diarreia, hemoptise, coriza e tosse produtora de fleuma (Marinho et al., 2020).

Até o momento, não existem medicamentos antivirais contra o SARS-CoV-2 e os principais tratamentos são de acordo com cada sintomas do paciente. A comunidade científica mundial tem adotado a estratégia de reposicionamento de fármacos na busca de medicamentos que sejam eficazes em curto período de tempo (Altay et al., 2020). Os métodos tradicionais de descoberta de medicamentos podem levar anos, o que não seria interessante para o momento (Pillaiyar et al., 2020). Além disso, as informações relacionadas a disponibilidade, farmacocinética, farmacodinâmica e toxidade, são uma vantagem importante nas pesquisas por medicamentos contra doenças (Zheng et al., 2018).

Alguns medicamentos têm sido relatados como possibilidade de tratamentos, entre eles: cloroquina, hidroxicloquina, remdesivir e ivermectina. Estudos demonstraram que a ivermectina inibe a replicação viral in vitro do SARS-CoV-2 (Caly et al., 2020), mas ainda não há testes clínicos para analisar a sua eficácia. Ainda com número pequeno de pacientes hospitalizados por COVID19 no estado grave, 36 dos 56 pacientes tiveram uma melhora clínica quando tratados com o remdesivir, entretanto o estudo não apresentou critérios bem definidos que para fornecer evidências clínicas suficientes para concluir a eficácia deste fármaco para a COVID-19 (Grein et al., 2020). A hidroxicloroquina e a cloroquina também foram testadas para o tratamento da COVID-19 e ambos os medicamentos conseguiram inibir a replicação viral do SARS-CoV-2 em estudos in vitro (Khuroo et al., 2020), mas não apresentaram eficácia comprovada em nenhum dos estudos clínicos (Ortiz-Prado et al., 2020). Outros estudos, mas dessa vez com pacientes, demonstraram que a cloroquina ou hidroxicloroquina não devem ser utilizadas contra o COVID-19 em estado grave, especialmente associada com azitromicina e oseltamivir (Borba et al., 2020). Além disso, outros trabalhos relataram a toxicidade cardíaca aguda provocada por doses de hidroxicloroquina e cloroquina (Della Porta et al., 2020). Diante isso, a pesquisa por medicamentos que possam ser eficazes contra o SARS-CoV-2 ainda é uma estratégia importante para o tratamento do coronavírus.

Compostos heterocíclicos aromáticos contendo átomos de nitrogênios, se destacam no campo da química medicinal pelo seu amplo potencial biológico, incluindo efeito antiviral (García et al., 2003; Sun et al., 2020), antibacteriano (Gonzaga et al., 2013), antifúngico (Thanh et al., 2019) e antiparasitário (Tahghighi et al., 2012). Além disso, derivados azólicos, especialmente o 1,2,3triazol pode ser facilmente sintetizado com baixo custo, através da reação click chemistry, reações que podem ser catalisadas por iodeto de cobre entre alcinos e azidas, com formação de um grupamento 1,2,3-triazólico (Tale et al., 2015). Estudos clínicos indicaram que o itraconazol, um fármaco derivado dos azóis, são seguros e bem tolerados sem efeitos colaterais graves em pacientes pediátricos (De Repentigny et al., 1998; Schmitt et al., 2001; Groll et al., 2002). Dessa forma, o estudo do potencial contra SARSCoV-2 de derivados azólicos se constitui de uma alternativa atrativa para o reposicionamento de fármacos com potencial sobre o novo coronavírus. Deste modo, medicamentos como: cetoconazol, fluconazol, itraconazol e voriconazol apresentam em sua estrutura química derivados de azóis (Moreira, 2010) e podem ser bons candidatos para inibir a replicação do SARS-CoV-2.

$\mathrm{Na}$ estratégia de reposicionamento ou descoberta de fármacos, o reconhecimento de alvos em sistemas biológicos tem-se um papel importante, pois ajudam no entendimento de propriedades como potência, afinidade e seletividade. Com isso, a protease do SARS-CoV-2, é um alvo atraente de medicamentos antivirais, pois são essenciais para a replicação do vírus (Báez-Santos et al., 2015). A protease do tipo 3c (3CL $\left.{ }^{\text {pro }}\right)$ do SARS-CoV-2, é essencial no processamento das poliproteínas, 16 proteínas não estruturais (NSPs). Outro alvo de medicamentos contra o COVID-19 é a proteína nucleocapsídeo viral (Proteína nucleocapsídeo viral), é responsável pela maior proporção de proteínas da estrutura viral, apresenta como função principal empacotar o genoma do RNA viral de genoma fita simples em um complexo de ribonucleoproteínas, chamado de capsídeo (Chang et al., 2014). A inibição desses alvos pode ser crucial para sobrevivência do vírus (Elzupir, 2020; Fu et al., 2020). Mediante a esse cenário de pandemia e a busca 
por medicamentos eficazes a um curto período de tempo, o presente estudo investigou as propriedades farmacocinéticas (ADME), físicos químicos e toxicidade dos medicamentos derivados de azóis. As análises de docking molecular dos fármacos que se mostraram mais promissores nas análises in silico frente a $3 \mathrm{CL}^{\text {pro }}$ e a proteína nucleocapsídeo viral do SARS-CoV-2.

\section{MATERIAL E MÉTODOS}

\section{Análise do Perfil Farmacocinético e in silico dos Fármacos}

As estruturas químicas dos fármacos cetoconazol, fluconazol, voriconazol e itraconazol (Figura 1) foram obtidas através do banco de dados PubChem (https://pubchem.ncbi.nlm.nih.gov/). A análise do perfil físico-químico e farmacocinético (ADME) foi realizada através do SwissADME e pkCSM.

Figura 1. Estrutura química dos fármacos Itraconazol (1), Cetoconazol (2), Fluconazol (3) e Voriconazol (4).

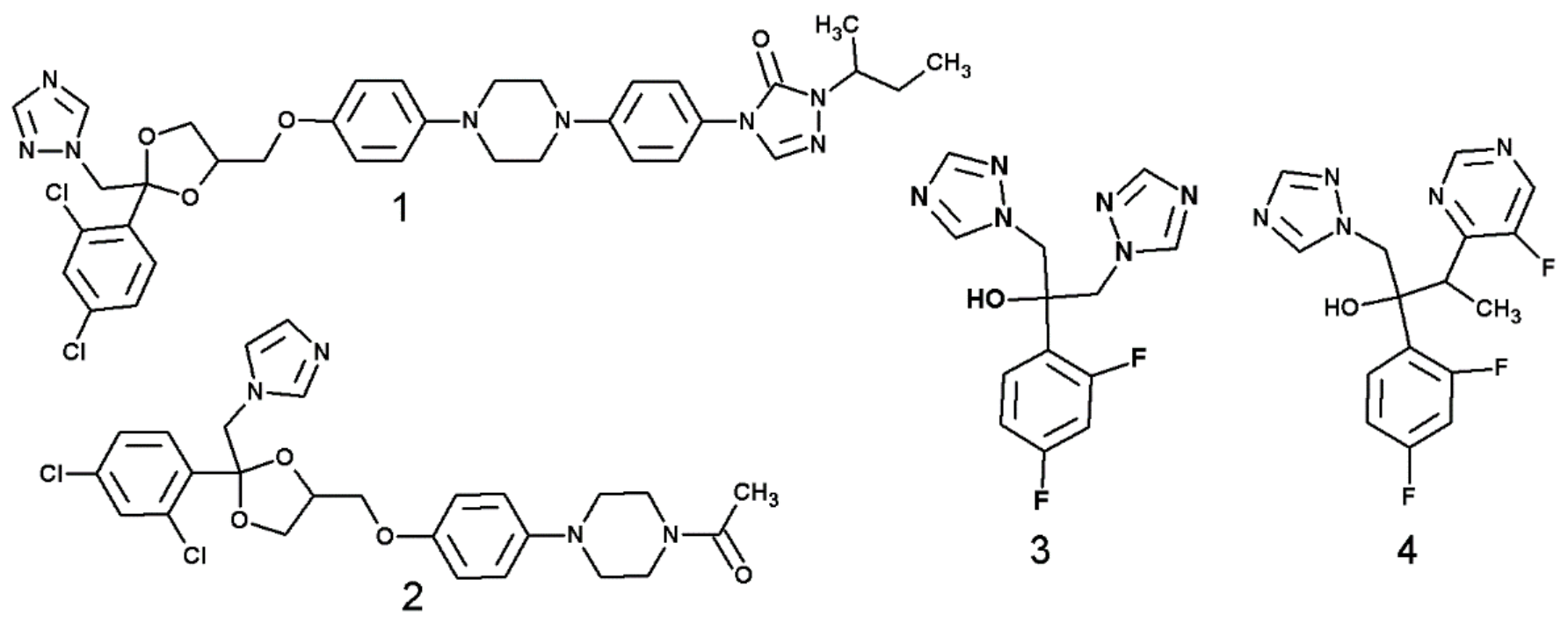

CONFRONTO DOS FÁRMACOS CETOCONAZOL, FLUCONAZOL, VORICONAZOL E ITRACONAZOL CONTRA ALVOS DO SARS-COV2 POR DOCKING MOLECULAR

As análises de interações molecular dos ligantes com as proteínas alvos foram realizadas através de ancoragem molecular usando software Autodock $4.2 \mathrm{e}$ $i$ GemDock 2.1. As estruturas das macromoléculas foram obtidas no protein data bank (PDB) (Berman et al., 2003). Foram selecionados 2 alvos comumente relacionados a ação de drogas: protease principal do SARS-CoV-2 (5R80) e proteína do nucleocapsídeo (6VYO).

Para as análises, as proteínas foram preparadas previamente conforme descrito por Velásquez et al. (2013). Inicialmente, os locais de ligações eram desconhecidos, assim foram realizados ajustes para o melhor encaixe individualmente do ligante-alvo. Para isso, a grade foi ajustada para toda a molécula e adequado o tamanho nas direções $\mathrm{x}, \mathrm{y}, \mathrm{z}$. O software forneceu as 10 melhores conformações estimadas de interação ligantealvo e assim foram selecionados os melhores valores de energia para continuar nesse estudo. As interações do complexo ligantes-alvo, foram analisadas e a renderização foi feita através do Discovery Studio 2020.

\section{RESULTADOS E DISCUSSÃO}

Os azóis são grupos farmacofóricos heterocíclicos contendo átomos de nitrogênios com alta atividade contra fungos, além disso esses grupos estão presentes em vários fármacos, como: cetoconazol, itraconazol, voriconazol e fluconazol (Melo et al., 2006; Salehi et al., 2020). Todos esses medicamentos citados anteriormente, são utilizados 
no tratamento com infecções fúngicas e estão disponíveis em formulações orais, entretanto o cetoconazol também pode ser encontrado em formulações tópicas (Martinez, 2006; Rezende et al., 2017). Para a busca de fármacos promissores contra a COVID-19, os medicamentos utilizado no tratamento precisam ser eficazes contra a SARS-CoV-2 (Elzupir, 2020), além disso devem apresentar boas propriedades farmacocinéticas, farmacodinâmica para serem administradas por via oral e baixa toxicidade (Daina et al., 2017; Vardhan e Sahoo, 2020). A administração por via oral é um critério de seleção particularmente importante de fármacos candidatos a tratamentos de doenças, essa via de administração é a mais simples, segura, baixo custo e facilmente autoadministradas (Stoner et al., 2004).

Para um fármaco ser administrado por via oral é necessário que a molécula possa apresentar um perfil de acordo com os parâmetros das regras de Lipinski ou regra dos cinco (Santos et al., 2018). A molécula deve ser constituída por até 5 doadores de ligações de hidrogênio, 10 aceitadores de ligações de hidrogênio, massa molecular no máximo $500 \mathrm{~g} / \mathrm{mol}$ e coeficiente de partição octanolágua (cLogP) entre 0 a 5 (Lipinski, 2000). Além de Lipinski, Veber et al. (2002) também estabeleceram parâmetros, como números de ligações rotativas inferiores a 10 e uma área de superfície polar (TPSA) menor que 140 A22. As moléculas que obedecem a esses parâmetros podem ter mais chances para serem facilmente absorvidas pelo trato gastrointestinal (Santos et al., 2018).

De acordo com as regras estabelecidas por Lipinski e Veber, os fármacos itraconazol e cetoconazol violam os parâmetros (Tabela 1). $\mathrm{O}$ itraconazol não apresentou peso molecular e número de ligações rotativas dentro dos parâmetros estabelecidos por Lipinski e Veber, já o cetoconazol também não apresentou peso molecular estabelecido pela regra dos cinco, os demais fármacos não violam nenhum dos parâmetros da regra dos cinco ou Veber. Moléculas que apresentam alto peso molecular podem não ser bem absorvidas pelo trato gastrointestinal, além disso compostos com peso molecular menor que 500 $\mathrm{g} / \mathrm{mol}$ são facilmente transportadas por proteínas (Mishra et al., 2017). Já as moléculas que apresentam valores de ligações rotativas maiores que 10 , tendem a rotacionar mais pondendo assim, reduzir o potencial de absorção, pois é comumente necessário a adoção de uma conformação fixa para interagir com o alvo (Chen et al., 2020; Corsini, 2020).

Outros parâmetros como $\operatorname{cLog}$ e TPSA são variáveis fisico-químicas úteis, pois estão relacionados às etapas que atecendem o efeito biológico: fase farmacêutica, farmacocinética e farmacodinâmica (Manadas et al., 2002). Na fase farmacêutica, o fármaco será desintegrado, na fase farmacocinética o fármaco será absorvido, distribuído, metabolizado e excretado, na fase farmacodinâmica haverá interação fármaco-receptor e assim haverá o efeito terapêutico (Pereira, 2007). Dessa forma, moléculas anfipáticas podem chegar melhor no efeito terapêutico. Com isso, o voriconazol apresenta cLogP de 2,40 e TPSA de 76,72 $\AA^{2}$, visto que cLogP próximo a 5 caracteriza a molécula como caráter lipofílico, já números próximos a 0 , caracteriza como hidrofílico e o TPSA é útil para indicar a área de polaridade da molécula (Sá et al., 2010).

Tabela 1. Análise da regra de Lipinski e Veber dos medicamentos itraconazol, fluconazol, voriconazol e cetoconazol

\begin{tabular}{lcccc}
\hline \multicolumn{1}{c}{ Propriedades } & Itraconazol & Fluconazol & Voriconazol & Cetoconazol \\
\hline HBA & 7 & 7 & 8 & 5 \\
HBD & 0 & 1 & 1 & 0 \\
cLogP & 4,74 & 0,88 & 2,40 & 3,55
\end{tabular}
MW (g/mol)
705,63
306,27
349,31
531,43

n-ROTB

TPSA $\left(\AA^{2}\right)$
11

104,70
5

5

76,72
8

81,65

(6.

72

69,06

Legenda. HBA: número de aceitadores de ligações de hidrogênio; HBD: número de doadores de hidrogênio; cLogP: logaritmo do coeficiente de partição entre octanol/água (lipofilicidade); MW: peso molecular; n-ROTB: número de 
ligações rotativas, TPSA: área de superfície polar.

A primeira etapa da farmacocinética é a absorção do fármaco, processo que depende de a capacidade dessa substância ultrapassar as barreiras biológicas como o trato gastrointestinal sob influência das características físicoquímicas de ambos (Souza et al., 2007). A análise da absorção revelou que todos os fármacos apresentaram bons valores preditivo de absorção gastrointestinal, e apenas voriconazol e cetoconazol apresentaram potencial para atravessar a barreira hematoencefálica (Tabela 2). Apesar do fármaco itraconazol violar parâmetros como massa molecular $(\leq 500 \mathrm{~g} / \mathrm{mol})$ e $\mathrm{n}$-ROTB $(\leq 10)$ e o cetoconazol também apresentar massa superior aos valores ideais estabelecidos na regra dos cinco, ambos os fármacos apresentam propriedades que contribuem para uma boa absorção, como o cLogP e o TPSA, características essenciais para absorção. Esse aspecto pode estar relacionado ao fato de que, mesmo violando as referidas regras, esses compostos possuem boa absorção pelo TGI (Daina, et al., 2017). Além disso, apenas o itraconazol e o voriconazol apresentaram perfil compatível para serem substrato da glicoproteína (P-gp). A P-gp é um transporte de efluxo responsável por expulsar as substâncias das células (Huber et al., 2010; Vilar et al., 2019). Um papel importante das P-gp é proteger o sistema nervoso central dos xenobióticos (Szakács et al., 2008) e as enzimas do citocromo P450 (CYP450) são responsáveis pelo metabolismo dos xenobióticos, que são substâncias químicas poluentes ao organismo humano (González e Guerra, 2014).

Dessa forma, é essencial conhecer as interações dos fármacos propostos sobre $\mathrm{o}$ citocromo $\mathrm{P} 450$ : CYP1A2, CYP2C9, CYP2D6, CYP3A4 e CYP2C19. Essas enzimas são responsáveis por metabolizar substâncias químicas, ao adicionar grupos químicos para ativar ou inativar os fármacos, a inibição dessas enzimas poderá causar interações medicamentosas (Audi e Pussi, 2000). Além disso, a inibição dessas enzimas irá ocorrer efeitos indesejados devido ao acúmulo do medicamento ou de seus metabólitos no corpo humano (Hollenberg, 2002). Todos os fármacos apresentaram potencial para inibir pelo menos uma das enzimas do CYP450, com destaque para itraconazol que apresentou potencial de inibir todas as cinco enzimas principais, já o cetoconazol inibe quatro das cinco enzimas, o fluconazol e o voriconazol inibem apenas uma das cinco enzimas. Sabendo disso, parte do fluconazol é excretada inalterada por via renal (Colombo e Guimarães, 2007), já o itraconazol é biotransformado no fígado, assim o fármaco e seus metabólitos são excretados diretamente na urina e nas fezes. O cetoconazol é metabolizado no fígado, eliminado pela bile e também por via renal (Pereira, 2007), o voriconazol é eliminado pela biotransformação das enzimas CYP450 e excretado na urina (Geist et al., 2013). No rim, o transportador de cátions orgânicos 2 renal (OCT2) é expresso na membrana basolateral do epitélio do túbulo proximal e estar envolvido na captação de muitos xenobióticos da corrente sanguínea para as células epiteliais renais. Apenas o cetoconazol mostrou potencial para ser OCT2 (Shikata et al., 2007).

Tabela 2. Farmacocinética e excreção dos fármacos itraconazol, fluconazol, voriconazol e cetoconazol

\begin{tabular}{|c|c|c|c|c|}
\hline Propriedades & Itraconazol & Fluconazol & Voriconazol & Cetoconazol \\
\hline BBB & Não & Não & Sim & Sim \\
\hline GIA & Alto & Alto & Alto & Alto \\
\hline P-Gp & Sim & Não & Sim & Não \\
\hline $\begin{array}{l}\text { CYP450 2C9 } \\
\text { inibidor }\end{array}$ & Sim & Não & Não & Sim \\
\hline $\begin{array}{c}\text { CYP450 } \\
\text { 2D6 inibidor }\end{array}$ & Sim & Não & Sim & Sim \\
\hline $\begin{array}{c}\text { CYP450 2C19 } \\
\text { inibidor }\end{array}$ & Sim & Sim & Não & Sim \\
\hline $\begin{array}{l}\text { CYP450 3A4 } \\
\text { inibidor }\end{array}$ & Sim & Não & Não & Sim \\
\hline
\end{tabular}




\begin{tabular}{ccccc}
\hline $\begin{array}{c}\text { CYP450 1A2 } \\
\text { inibidor }\end{array}$ & Sim & Não & Não & Não \\
DT $(\log \mathbf{~ m L / m i n} / \mathbf{k g})$ & 0,056 & 0,29 & 0,194 & 0,601 \\
OCT2 & Não & Não & Não & Sim \\
\hline
\end{tabular}

Legenda. BBB: barreira hematoencefálica; GIA: absorção gastrointestinal; P-gp: glicoproteína de permeabilidade. Metabolismo: CYP450: Enzima do Citocromo P450. Excreção: DT: Depuração total; OCT2: Substrato transportador de cátions orgânicos 2 renal.

Conhecer a toxicidade de uma substância é útil e importante para a busca de novos medicamentos ou reposicionamento de fármacos. Dessa forma, o conhecimento das propriedades tóxicas associado a conhecer a estrutura química do fármaco, pode reduzir os efeitos colaterais (Banerjee et al., 2018). A predição do teste de AMES é utilizada para determinar o potencial mutagênico e possível potência carcinogênico das moléculas (Gunther et al., 2017). Nenhum dos fármacos apresentaram potencial para serem mutagênicos (Tabela 3). A predição da inibição dos genes humanos éter-a-gogo I e II (hERG I e II), codifica a subunidade formadora de poros dos canais de potássio, apresenta um papel importante na repolarização dos potenciais de ação cardíaca (Lamothe et al., 2016). A inibição desses canais é principal causa para o desenvolvimento da síndrome do QT longo, levando à arritmia ventricular fatal (Priest et al., 2008; Maior et al., 2011). Nenhum dos fármacos apresentaram potencial para inibição do hERG I, já para o hERG II os fármacos itraconazol e cetoconazol apresentaram potencial para causar alguma interação com os canais de potássio. O SARS-CoV-2 infecta as células hospedeiras por meio de receptores da enzima conversora de angiotensina 2, levando à pneumonia, além disso poderá causar lesão miocárdica aguda e danos crônicos ao sistema cardiovascular (Zheng et al., 2020). Dessa forma, para um possível tratamento, os fármacos itraconazol e cetoconazol não poderiam ser administrados a pacientes com problemas cardíacos e com COVID-19 por conta do potencial de inibição dos hERG II, pois essa intervenção farmacológica poderia agravar o quadro clínico do paciente. Recentemente, a hidroxicloroquina e azitromicina foram drogas utilizadas com o objetivo de tratar pacientes com COVID-19 e foi observado que esses medicamentos prolonga o intervalo do QT (Voisin et al., 2020).

Tabela 3. Predição de toxicidade dos fármacos itraconazol, fluconazol, voriconazol e cetoconazol

\begin{tabular}{ccccc}
\hline Propriedades & Itraconazol & Fluconazol & Voriconazol & Cetoconazol \\
\hline AMES & Não & Não & Não & Não \\
hERG I & Não & Não & Não & Não \\
hERG II & Sim & Não & Não & Não \\
SS & Não & Não & Não & 2,84 \\
DL50 \\
mol/Kg
\end{tabular}

Legenda. AMES: predição potencial mutagênico; hERG I / II: predição de inibição ao gene ether-a-go-go; SS Sensibilização da pele; $\mathrm{DL}_{50}$ : dose letal em $50 \%$.

Após a predição de ADME, é importante investigar a interação molecular dos fármacos que apresentaram melhores valores nas análises in silico. $\mathrm{O}$ fármaco itraconazol viola parâmetros da regra dos cinco em massa molecular $(\leq 500 \mathrm{~g} / \mathrm{mol})$ e número de ligações rotativas $(\leq 10)$, já o cetoconazol também apresenta massa 
molecular superior aos valores ideias, ambos os fármacos inibem as enzimas do CYP450, além disso apresentam potencial para interagir com os genes dos canais de potássio. Já os fármacos fluconazol e voriconazol não violam a regra de Lipinski, apresentaram potencial para inibir apenas uma enzima do CYP450 e não apresentam valores preditivos de interações com os genes dos canais de potássio. Dessa forma investigamos a interação do complexo ligante-receptor dos fluconazol e voriconazol frente a alvos do SARS-CoV-2.
As análises de interações receptor-ligante mostraram valores totais de energia para a $3 \mathrm{CL}^{\text {pro }} \mathrm{de}$ 86,81 e $-88,01 \mathrm{kcal} / \mathrm{mol}$ para fluconazol e voriconazol, respectivamente. Já para proteína nucleocapsídeo viral foram de $-88,18$ e $-106,14 \mathrm{kcal} / \mathrm{mol}$ para fluconazol e voriconal, respectivamente. Além disso, os fármacos apresentaram interação com os alvos da SARS-CoV-2 (Tabela 4), mostrando que os fármacos podem interagir com a proteína nucleocapsídeo viral e $3 \mathrm{CL}^{\text {pro }}$.

Tabela 4. Perfil de interação entre os fármacos com alvos da SARS-CoV-2

\begin{tabular}{|c|c|c|c|c|}
\hline & \multicolumn{2}{|c|}{ Fluconazol } & \multicolumn{2}{|c|}{ Voriconazol } \\
\hline & $3 C L^{\text {pro }}$ & $\begin{array}{c}\text { Proteína } \\
\text { nucleocapsídeo } \\
\text { viral }\end{array}$ & $3 C L^{\text {pro }}$ & $\begin{array}{c}\text { Proteína } \\
\text { nucleocapsídeo } \\
\text { viral }\end{array}$ \\
\hline EL (kcal/mol) & -5.08 & -3.95 & -5.52 & -4.85 \\
\hline LE & -0.23 & -0.18 & -0.22 & -0.19 \\
\hline $\mathrm{CI}(\boldsymbol{\mu M})$ & 189.6 & 1027 & 90.23 & 277.11 \\
\hline EnI & -6.87 & -5.74 & -7.31 & -6.64 \\
\hline VDW-HB-DE & -6.86 & -5.7 & -7.22 & -6.73 \\
\hline EEc & -0.01 & -0.04 & -0.09 & -0.09 \\
\hline TI & -1.79 & -2.06 & -1.75 & -2.44 \\
\hline EnT & 1.79 & 1.79 & 1.79 & 1.79 \\
\hline EnC & -1.79 & -2.06 & -1.75 & -2.44 \\
\hline clRMS & 0.0 & 0.0 & 0.0 & 0.0 \\
\hline DifRMS & 23.37 & 47.11 & 8.13 & 51.16 \\
\hline Rseed 1 e Rseed 2 & Nenhum & Nenhum & Nenhum & Nenhum \\
\hline
\end{tabular}

Legenda. EL: energia de ligação; LE: eficiência do ligante; $\mathrm{CI}(\mu \mathrm{M})$ : constante de inibição; EnI: Energia Intermolecular; VDW-HB-DE: Vdw-hb-dissolve energia; Eec: energia eletrostática; TI: Total interno; EnT: energia de torção; EnC: Energia não consolidada; clRMS: valor quadrado médio da raiz do valor; DifRMS: diferença de RMS entre coordenadas de conformação de corrente e estrutura de referência atual; Rseed 1 e Rseed 2: as sementes de números aleatórios específicos usadas para a execução de encaixe da conformação atual.

O fluconazol apresentou potencial de interação com ambos os alvos do SARS-CoV-2 e valores de energia mais favorável para a $3 \mathrm{CL}^{\text {pro }}$, do que para a proteína nucleocapsídeo viral. Dessa forma, o fluconazol pode 


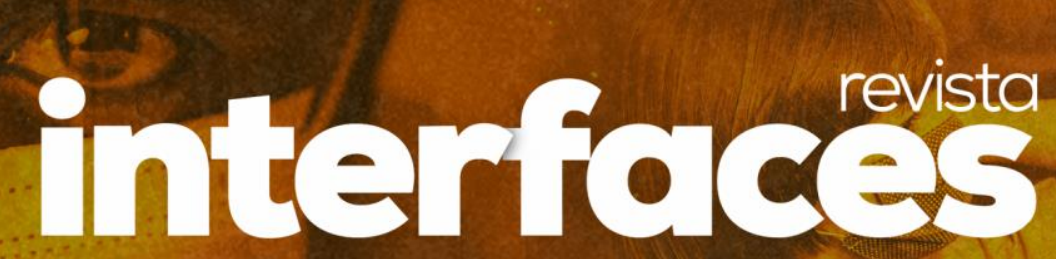

interagir com ambas as proteínas, com valores de afinidade de ligação de -5.08 e $-3.95 \mathrm{kcal} / \mathrm{mol}$, além disso a constante de inibição foi de 189.6 e $1027 \mu \mathrm{M}$, para a $3 \mathrm{CL}^{\text {pro }}$ e proteína nucleocapsídeo viral, respectivamente. Já o voriconazol também demonstrou interação com ambos os alvos, apresentou valores de afinidade de ligação de -5.52 e $-4.85 \mathrm{kcal} / \mathrm{mol}$ e constante de inibição de 90.23 e $277.11 \mu \mathrm{M}$, para a $3 \mathrm{CL}^{\text {pro }}$ e proteína nucleocapsídeo viral, respectivamente. Deste modo, o voriconazol, mostrou maior pontecial de ligação e interação molecular com ambos os alvos do SARS-CoV-2, quando comparado ao fluconazol. Dessa forma, foi investigado quais as ligações estariam relacionadas na ligação entre receptorligante.

$\mathrm{O}$ fármaco voriconazol interage com a $3 \mathrm{CL}^{\text {pro }}$ formando ligações do tipo van der Waals, alquil, Pi Sigma, Pi- Alquil, ligação de hidrogênio e carbono (Figura 2). $\mathrm{O}$ anel heterocíclico contendo dois átomos de nitrogênio e quatro átomos de carbonos do voriconazol interagiu com aminoácidos MET6, PHE8, ARG298, já o triazol mostrou interação com ILE152 e VAL303. A interação receptor-ligante do voriconazol com a proteína nucleocapsídeo viral do SARS-CoV-2, mostrou ligações do tipo van der Waals, Pi - Sigma, Pi- Alquil, ligação com halogênio (flúor), ligação de hidrogênio convencional e carbono (Figura 3). O anel aromático apresentou potencial para interagir com aminoácidos ALA173 e LEU167, além disso o átomo de flúor da posição orto fez ligação com aminoácido GLN163, o flúor da posição para mostrou ligações com ALA173, LEU161 e PRO162.

Figura 2. Interações do voriconazol sobre a $3 \mathrm{CL}^{\text {pro }}$. Imagem A e B, compostos no sítio de ligação - Interações do Voriconazol sobre a $3 \mathrm{CL}^{\text {pro }}$
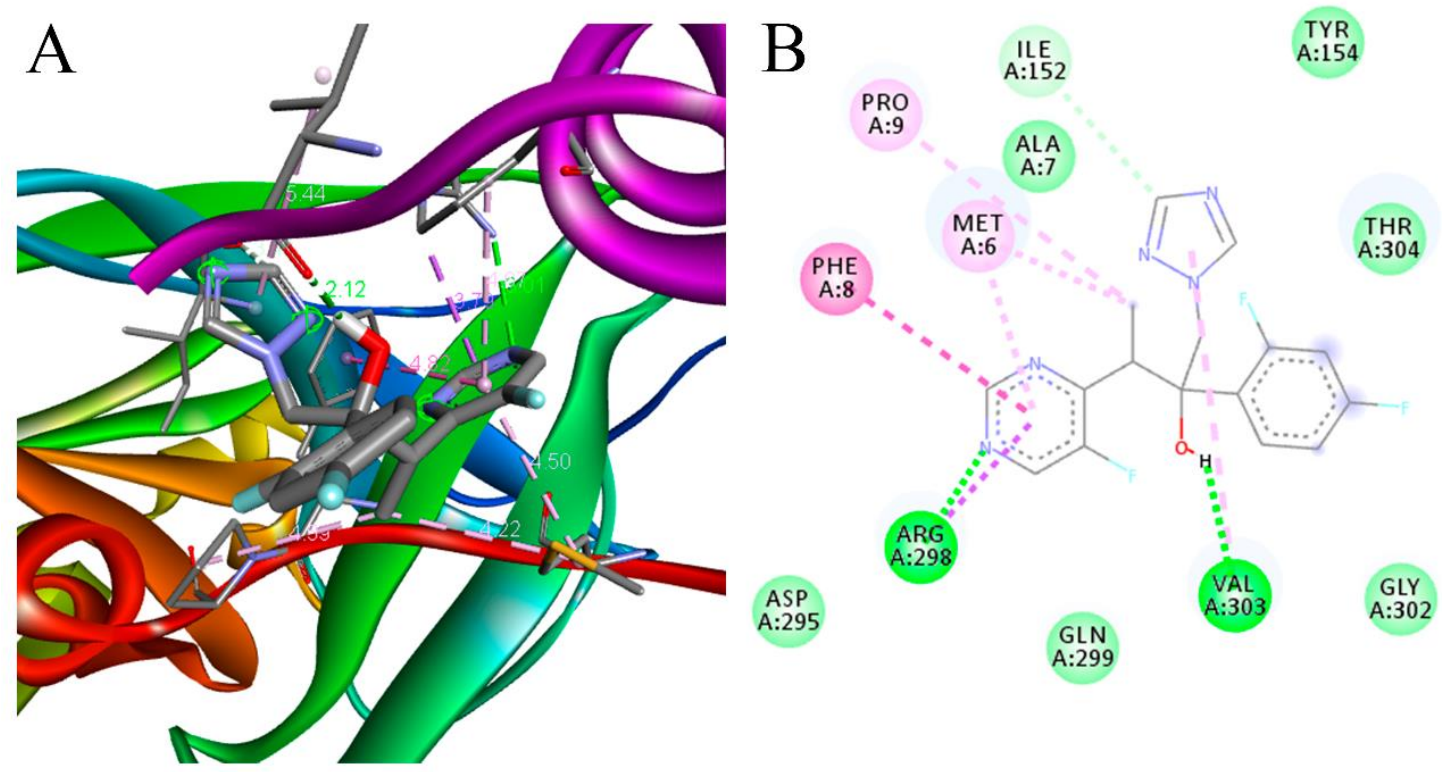

Interações

Ligação de hidrogênio convencional Ligação de carbono e hidrogênio

Pi-Pi em forma de T

Estudos demonstraram que o bloqueio da função de clivagem da $3 \mathrm{CL}^{\text {pro }}$ pode-se inibir a replicação do vírus, tornando está enzima um dos alvos mais atraente para o tratamento do COVID-19 (Sisay, 2020). Assim, a 3CL ${ }^{\text {pro }}$ tem como função clivar a poliproteína em locais diferentes para gerar várias proteínas não estruturais que são importantes para a replicação viral (Anand et al., 2003). Além da $3 \mathrm{CL}^{\text {pro }}$, a proteína nucleocapsídeo viral chama atenção dos pesquisadores pela sua ligação ao RNA van der Waals

Pi-Sigma

Alquil

Pi-Alquil

multifuncional, que é essencial para a transcrição e replicação do RNA viral. Além disso, ela desempenha outros papéis importantes como na formação de ribonucleoproteínas helicoidais durante o empacotamento do genoma do RNA viral, regula a síntese do RNA viral na replicação / transcrição e modula o metabolismo celular infectado (Stohlman et al., 1988; Nelson et al., 2000). A inibição da enzima $3 C^{\text {pro }}$ e a proteína nucleocapsídeo viral pode ser essencial no desenvolvimento de novos 


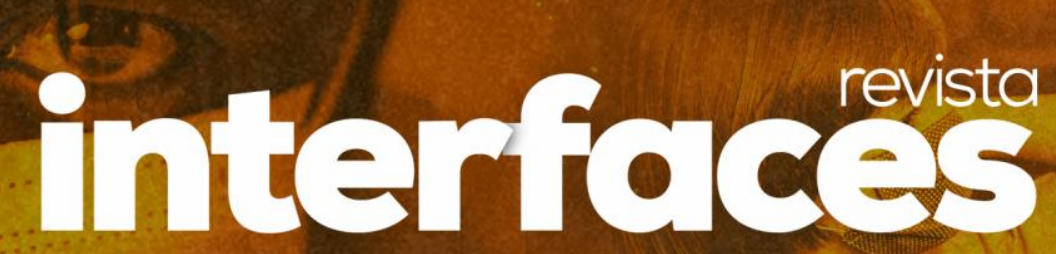

fármacos contra o COVID-19 (Ghosh et al., 2007; Kang et

al., 2020).

Figura 3. Interações do voriconazol sobre a Proteína nucleocapsídeo viral. Legenda: Imagem A e B, tipos de ligações que são produzidas no sítio de ligação - Interações da Voriconazol sobre proteína do nucleocapsídeo viral.
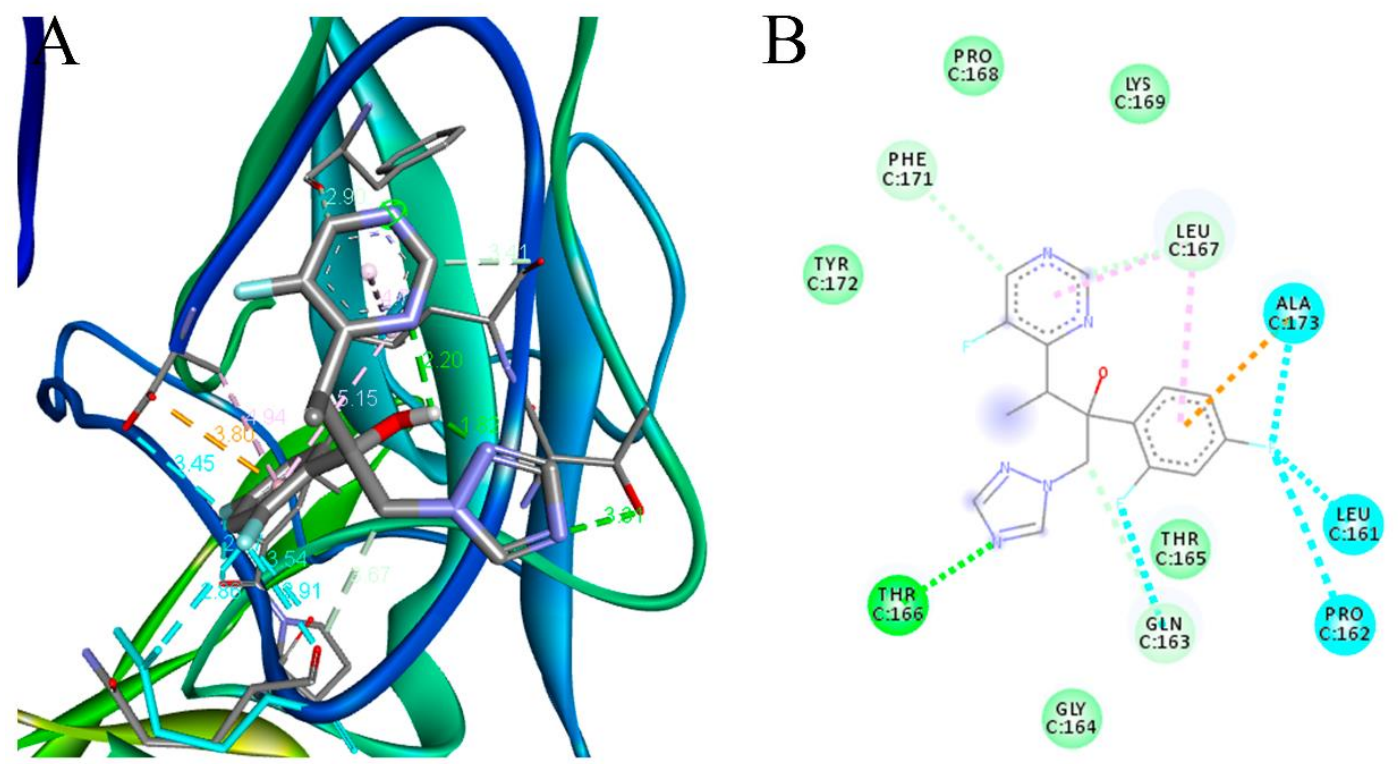

\section{Interações}

Ligação de hidrogênio convencional

Ligação de carbono e hidrogênio

van der Waals

Halogênio (flúor)

Pi-Anion

Pi-Alquil
O fluconazol apresenta em sua estrutura química 2 grupos triazólicos cada um ligado a um carbono primário, já o voriconazol contém em sua estrutura química um grupo triazol, também ligado a um carbono primário (Aher et al., 2009). Estudos avaliaram o potencial antiviral de moléculas contendo o grupo triazol e observaram atividade antiviral em alguns dos compostos testados (He et al., 2014). Em comparação com ambas as estruturas químicas dos fármacos fluconazol e voriconazol, observa-se uma estrutura semelhante (Figura 4), visto que ambas as moléculas apresentam triazol, anel aromático fazendo ligação com dois átomos de flúor na posição orto e para, além disso uma hidroxila ligada a um carbono terciário (Aher et al., 2009). O efeito semelhante de interação molecular dos fármacos frente aos alvos do SARS-CoV-2 deve-se ao fato da semelhança estrutural de ambas estruturas químicas. Com isso, a presença de um único triazol pode ter sido ideal para uma melhor interação do complexo receptor-ligante, dessa forma o voriconazol apresentou melhores valores interações com ambos os alvos do SARS-CoV-2 comparando com o fluconazol. 
Figura 4. Semelhança estrutural dos fármacos fluconazol (1) e voriconazol (2)

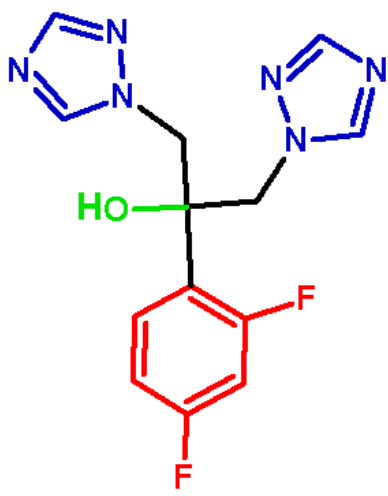

1

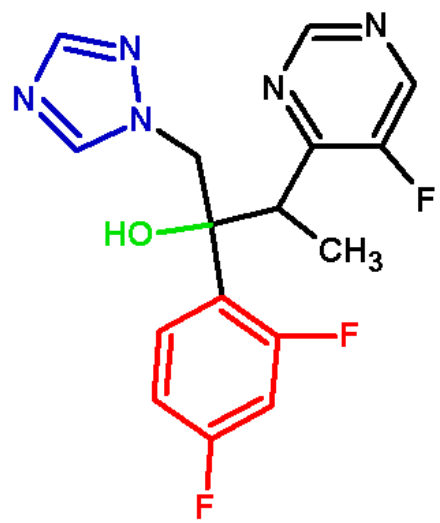

2

\section{CONCLUSÃO}

Em nosso estudo, foi possível concluir que o fármaco itraconazol apresentou potencial para inibir todas as enzimas do citocromo P450, já o cetoconazol mostrouse potencial para interagir com quatro das cinco enzimas do CYP450. Além disso, ambos os fármacos mencionados violam as regras de Lipinski e Veber, caracterizando assim como moléculas com baixo potencial para biodisponibilidade oral. Os fármacos fluconazol e voriconazol não violaram a regra dos cinco e Veber, por isso foram feitas análises de docking molecular desses fármacos. Assim, ambos os fármacos apresentaram valores de energias favoráveis, havendo potencial para interagir com a proteína nucleocapsídeo viral e a $3 \mathrm{CL}^{\text {pro }} \mathrm{do}$ SAR-CoV-2. Dessa forma, sugere-se análise de dinâmica molecular, estudos mais aprofundados in vitro e in vivo para investigar as atividades desses fármacos frente ao vírus.

\section{AGRADECIMENTOS}

Fundação de Amparo à Ciência e Tecnologia do Estado de Pernambuco (FACEPE), Laboratório de Biologia Celular de Patógenos, Instituto Aggeu Magalhães.

\section{REFERÊNCIAS}

AHER, N. G. et al. Synthesis and antifungal activity of 1, 2, 3triazole containing fluconazole analogues. Bioorganic \& medicinal chemistry letters, v. 19, n. 3, p. 759-763, 2009. ISSN 0960-894X.

ALTAY, O. et al. Current status of COVID-19 therapies and drug repositioning applications. Iscience, p. 101303, 2020. ISSN 2589-0042.

ANAND, K. et al. Coronavirus main proteinase (3CLpro) structure: basis for design of anti-SARS drugs. Science, v. 300, n. 5626, p. 1763-1767, 2003. ISSN 0036-8075.

APRESENTAÇÕES, F. F. E.; ORAL, V. MODELO DE BULA DO PROFISSIONAL DE SAÚDE. CEP, v. 4566, p. 905,

AUDI, E. A.; PUSSI, F. D. Isoenzimas do CYP450 e biotransformação de drogas. Acta Scientiarum. Biological
Sciences, v. 22, p. 599-604, 2000. ISSN 1807-863X.

BÁEZ-SANTOS, Y. M.; JOHN, S. E. S.; MESECAR, A. D. The SARS-coronavirus papain-like protease: structure, function and inhibition by designed antiviral compounds. Antiviral research, v. 115, p. 21-38, 2015. ISSN 0166-3542.

BANERJEE, P. et al. ProTox-II: a webserver for the prediction of toxicity of chemicals. Nucleic acids research, v. 46, n. W1, p. W257-W263, 2018. ISSN 0305-1048.

BERMAN, H.; HENRICK, K.; NAKAMURA, H. Announcing the worldwide protein data bank. Nature Structural \& Molecular Biology, v. 10, n. 12, p. 980-980, 2003. ISSN 15459985.

BORBA, M. G. S. et al. Effect of high vs low doses of chloroquine diphosphate as adjunctive therapy for patients 
hospitalized with severe acute respiratory syndrome coronavirus 2 (SARS-CoV-2) infection: a randomized clinical trial. JAMA network open, v. 3, n. 4, p. e208857-e208857, 2020.

CALY, L. et al. The FDA-approved drug ivermectin inhibits the replication of SARS-CoV-2 in vitro. Antiviral research, p. 104787, 2020. ISSN 0166-3542.

CHANG, C.-K. et al. The SARS coronavirus nucleocapsid protein-forms and functions. Antiviral research, v. 103, p. 3950, 2014. ISSN 0166-3542.

CHEN, X. et al. Analysis of the Physicochemical Properties of Acaricides Based on Lipinski's Rule of Five. Journal of Computational Biology, 2020. ISSN 1557-8666.

COLOMBO, A. L.; GUIMARÃES, T. Candidúria: uma abordagem clínica e terapêutica. Revista da Sociedade Brasileira de Medicina Tropical, v. 40, n. 3, p. 332-337, 2007. ISSN 0037-8682.

CORSINI, J. D. F. Bioinformática estrutural aplicada a busca por novos compostos contra os alvos moleculares p53-Y220C, MDM2, BIRC7, em câncer. 2020.

DAINA, A.; MICHIELIN, O.; ZOETE, V. SwissADME: a free web tool to evaluate pharmacokinetics, drug-likeness and medicinal chemistry friendliness of small molecules. Scientific reports, v. 7, p. 42717, 2017. ISSN 2045-2322.

DE REPENTIGNY, L. DE et al. Repeated-dose pharmacokinetics of an oral solution of itraconazole in infants and children. Antimicrobial agents and chemotherapy, v. 42, n. 2, p. 404-408, 1998. ISSN 0066-4804.

DELLA PORTA, A. et al. Acute chloroquine and hydroxychloroquine toxicity: A review for emergency clinicians. The American Journal of Emergency Medicine, 2020. ISSN 0735-6757.

ELZUPIR, A. O. Inhibition of SARS-CoV-2 main protease 3CLpro by means of $\alpha$-ketoamide and pyridone-containing pharmaceuticals using in silico molecular docking. Journal of Molecular Structure, p. 128878, 2020. ISSN 0022-2860.

FU, J. et al. Identification of a novel linear B-cell epitope on the nucleocapsid protein of porcine deltacoronavirus. International Journal of Molecular Sciences, v. 21, n. 2, p. 648, 2020.

FURUKAWA, N. W.; BROOKS, J. T.; SOBEL, J. Evidence supporting transmission of severe acute respiratory syndrome coronavirus 2 while presymptomatic or asymptomatic. Emerging infectious diseases, v. 26, n. 7, 2020.

GARCÍA, C. C.; CANDURRA, N. A.; DAMONTE, E. B. Differential inhibitory action of two azoic compounds against arenaviruses. International journal of antimicrobial agents, v. 21, n. 4, p. 319-324, 2003. ISSN 0924-8579.
GEIST, M. J. et al. Steady-state pharmacokinetics and metabolism of voriconazole in patients. Journal of Antimicrobial Chemotherapy, v. 68, n. 11, p. 2592-2599, 2013. ISSN 1460-2091.

GHOSH, A. K. et al. Structure-based design, synthesis, and biological evaluation of peptidomimetic SARS-CoV 3CLpro inhibitors. Bioorganic \& medicinal chemistry letters, v. 17, n. 21, p. 5876-5880, 2007. ISSN 0960-894X.

GONZAGA, D. et al. Recent advances in the synthesis of new antimycobacterial agents based on the $1 \mathrm{H}-1,2,3$-triazoles. Current topics in medicinal chemistry, v. 13, n. 22, p. 28502865, 2013. ISSN 1568-0266.

GONZÁLEZ, J. C. R.; GUERRA, I. R. El sistema citocromo P450 y el metabolismo de xenobióticos. Revista Cubana de Farmacia, v. 48, n. 3, p. 495-507, 2014. ISSN 1561-2988.

GREIN, J. et al. Compassionate use of remdesivir for patients with severe Covid-19. New England Journal of Medicine, v. 382 , n. 24, p. 2327-2336, 2020. ISSN 0028-4793.

GROLL, A. H. et al. Safety, pharmacokinetics, and pharmacodynamics of cyclodextrin itraconazole in pediatric patients with oropharyngeal candidiasis. Antimicrobial agents and chemotherapy, v. 46, n. 8, p. 2554-2563, 2002. ISSN 0066-4804.

GUNTHER, W. C. et al. Resolution of contradiction between in silico predictions and Ames test results for four pharmaceutically relevant impurities. Regulatory Toxicology and Pharmacology, v. 91, p. 68-76, 2017. ISSN 0273-2300.

HE, Y.-W. et al. 1, 2, 3-Triazole-containing derivatives of rupestonic acid: click-chemical synthesis and antiviral activities against influenza viruses. European Journal of Medicinal Chemistry, v. 76, p. 245-255, 2014. ISSN 0223-5234.

HOLLENBERG, P. F. Characteristics and common properties of inhibitors, inducers, and activators of CYP enzymes. Drug metabolism reviews, v. 34, n. 1-2, p. 17-35, 2002. ISSN 03602532.

HUBER, P. C.; MARUIAMA, C. H.; ALMEIDA, W. P. Glicoproteína-P, resistência a múltiplas drogas (MDR) e relação estrutura-atividade de moduladores. Quim. Nova, p. 21482154, 2010.

KANG, S. et al. Crystal structure of SARS-CoV-2 nucleocapsid protein RNA binding domain reveals potential unique drug targeting sites. Acta Pharmaceutica Sinica B, 2020. ISSN 2211-3835

KHUROO, M. S.; SOFI, A. A.; KHUROO, M. Chloroquine and Hydroxychloroquine in Coronavirus Disease 2019 (COVID19). Facts, Fiction \& the Hype. A Critical Appraisal. International Journal of Antimicrobial Agents, p. 106101, 2020. ISSN 0924-8579. 
LAMOTHE, S. M. et al. The human ether-a-go-go-related gene (hERG) potassium channel represents an unusual target for protease-mediated damage. Journal of Biological Chemistry, v. 291, n. 39, p. 20387-20401, 2016. ISSN 0021-9258.

LIPINSKI, C. A. Drug-like properties and the causes of poor solubility and poor permeability. Journal of pharmacological and toxicological methods, v. 44, n. 1, p. 235-249, 2000. ISSN 1056-8719.

MAIOR, A. S. et al. Canais iônicos de potássio associados à síndrome do QT longo adquirido. Rev Bras Cardiol, v. 24, n. 1, p. 42-51, 2011.

MANADAS, R.; PINA, M. E.; VEIGA, F. A dissolução in vitro na previsão da absorção oral de fármacos em formas farmacêuticas de liberação modificada. Revista Brasileira de Ciências Farmacêuticas, v. 38, n. 4, p. 375-399, 2002. ISSN 1516-9332.

MARINHO, E. M. et al. Virtual screening based on molecular docking of possible inhibitors of Covid-19 main protease. Microbial Pathogenesis, p. 104365, 2020. ISSN 0882-4010.

MARTINEZ, R. Atualização no uso de agentes antifúngicos. Jornal brasileiro de pneumologia, v. 32, n. 5, p. 449-460, 2006. ISSN 1806-3713.

MELO, J. O. et al. Heterociclos 1, 2, 3-triazólicos: histórico, métodos de preparação, aplicações e atividades farmacológicas. Química Nova, v. 29, n. 3, p. 569-579, 2006. ISSN 0100-4042.

MISHRA, S. S. et al. Computational Prediction of Pharmacokinetic, Bioactivity and Toxicity Parameters of Some Selected Anti arrhythmic Agents. The Pharmaceutical and Chemical Journal, v. 4, n. 5, p. 143-146, 2017.

MOREIRA, M. I. D. M. C. G. Azóis: farmacologia e interacções medicamentosas. 2010. [sn]

NELSON, G. W.; STOHLMAN, S. A.; TAHARA, S. M. High affinity interaction between nucleocapsid protein and leader/intergenic sequence of mouse hepatitis virus RNA. Journal of General Virology, v. 81, n. 1, p. 181-188, 2000. ISSN 0022-1317.

ORTIZ-PRADO, E. et al. Clinical, molecular and epidemiological characterization of the SARS-CoV2 virus and the Coronavirus disease 2019 (COVID-19), a comprehensive literature review. Diagnostic Microbiology and Infectious Disease, p. 115094, 2020. ISSN 0732-8893.

PEREIRA, D. G. Importância do metabolismo no planejamento de fármacos. Química nova, v. 30, n. 1, p. 171-177, 2007. ISSN 0100-4042.

PILLAIYAR, T. et al. A medicinal chemistry perspective of drug repositioning: Recent advances and challenges in drug discovery. European Journal of Medicinal Chemistry, p. 112275, 2020. ISSN 0223-5234.

PRIEST, B.; BELL, I. M.; GARCIA, M. Role of hERG potassium channel assays in drug development. Channels, v. 2, n. 2, p. 87-93, 2008. ISSN 1933-6950.

RANSING, R. et al. Mental Health Interventions during the COVID-19 Pandemic: A Conceptual Framework by Early Career Psychiatrists. Asian Journal of Psychiatry, v. 51, p. 102085-102085, 2020. ISSN 1876-2026.

REZENDE, C. et al. Mecanismos de ação dos antifúngicos. Rev Unifev Ciência Tecnol, v. 2, p. 222-36, 2017.

SÁ, M. M. D.; PASQUALOTO, K. F. M.; RANGEL-YAGUI, C. D. O. A 2D-QSPR approach to predict blood-brain barrier penetration of drugs acting on the central nervous system. Brazilian Journal of Pharmaceutical Sciences, v. 46, n. 4, p. 741-751, 2010. ISSN 1984-8250.

SALEHI, Z. et al. Comparison of in vitro antifungal activity of novel triazoles with old antifungal agents against dermatophyte species caused tinea pedis. Journal de Mycologie Médicale, p. 100935, 2020. ISSN 1156-5233.

SANTOS, V. L. D. A.; GONSALVES, A. D. A.; ARAÚJO, C. R. M. DIDACTIC APPROACH FOR THE DEVELOPMENT OF BIOATIVE MOLECULES: LIPINSKI'S FIVE-RULE AND PREPARATION OF 1, 3, 4-OXADIAZOL HETEROCYLIC IN DOMESTIC MICROWAVE OVEN. Química Nova, v. 41, n. 1, p. 110-115, 2018. ISSN 0100-4042.

SCHMITT, C. et al. Pharmacokinetics of itraconazole oral solution in neutropenic children during long-term prophylaxis. Antimicrobial agents and chemotherapy, v. 45, n. 5, p. 1561 1564, 2001. ISSN 0066-4804.

SERAFIN, M. B. et al. Drug repositioning an alternative for the treatment of coronavirus COVID-19. International Journal of Antimicrobial Agents, p. 105969, 2020. ISSN 0924-8579.

SHIKATA, E. et al. Human organic cation transporter (OCT1 and OCT2) gene polymorphisms and therapeutic effects of metformin. Journal of human genetics, v. 52, n. 2, p. 117-122, 2007. ISSN 1435-232X.

SISAY, M. 3CLpro inhibitors as a potential therapeutic option for COVID-19: Available evidence and ongoing clinical trials. Pharmacological research, v. 156, p. 104779, 2020.

SOUZA, J. D.; FREITAS, Z. M. F.; STORPIRTIS, S. Modelos in vitro para determinação da absorção de fármacos e previsão da relação dissolução/absorção. Revista Brasileira de Ciências Farmacêuticas, v. 43, n. 4, p. 515-527, 2007. ISSN 1516-9332.

STOHLMAN, S. et al. Specific interaction between coronavirus leader RNA and nucleocapsid protein. Journal of virology, v. 62, n. 11, p. 4288-4295, 1988. ISSN 0022-538X. 
STONER, C. L. et al. Integrated oral bioavailability projection using in vitro screening data as a selection tool in drug discovery. International journal of pharmaceutics, v. 269, n. 1, p. 241-249, 2004. ISSN 0378-5173.

SUN, L. et al. Design, synthesis and structure-activity relationships of 4-phenyl-1H-1, 2, 3-triazole phenylalanine derivatives as novel HIV-1 capsid inhibitors with promising antiviral activities. European Journal of Medicinal Chemistry, v. 190, p. 112085, 2020. ISSN 0223-5234.

SZAKÁCS, G. et al. The role of ABC transporters in drug absorption, distribution, metabolism, excretion and toxicity (ADME-Tox). Drug discovery today, v. 13, n. 9-10, p. 379393, 2008. ISSN 1359-6446.

TAHGHIGHI, A. et al. Synthesis and anti-leishmanial activity of 5-(5-nitrofuran-2-yl)-1, 3, 4-thiadiazol-2-amines containing $\mathrm{N}-[(1$-benzyl-1H-1, 2, 3-triazol-4-yl) methyl] moieties. European journal of medicinal chemistry, v. 50, p. 124-128, 2012. ISSN 0223-5234.

TALE, R. H.; GOPULA, V. B.; TORADMAL, G. K. 'Click'ligand for 'click'chemistry:(1-(4-methoxybenzyl)-1-H1, 2, 3-triazol-4-yl) methanol (MBHTM) accelerated coppercatalyzed [3+2] azide-alkyne cycloaddition (CuAAC) at low catalyst loading. Tetrahedron letters, v. 56, n. 43, p. 5864 5869, 2015. ISSN 0040-4039.

THANH, N. D. et al. Efficient click chemistry towards novel 1H-1， 2, 3-triazole-tethered 4H-chromene- d-glucose conjugates: Design, synthesis and evaluation of in vitro antibacterial, MRSA and antifungal activities. European Journal of Medicinal Chemistry, v. 167, p. 454-471, 2019. ISSN 0223-5234.

VARDHAN, S.; SAHOO, S. K. In silico ADMET and molecular docking study on searching potential inhibitors from limonoids and triterpenoids for COVID-19. arXiv preprint arXiv:2005.07955, 2020.

VEBER, D. F. et al. Molecular properties that influence the oral bioavailability of drug candidates. Journal of medicinal chemistry, v. 45, n. 12, p. 2615-2623, 2002. ISSN 0022-2623.

VELÁSQUEZ, M. et al. Método acoplado Autodock-PM6 para seleccionar la mejor pose en estudios de Acoplamiento Molecular. Revista Colombiana de Química, v. 42, n. 1, 2013. ISSN 0120-2804.

VILAR, S.; SOBARZO-SÁNCHEZ, E.; URIARTE, E. In silico prediction of P-glycoprotein binding: Insights from molecular docking studies. Current medicinal chemistry, v. 26, n. 10, p. 1746-1760, 2019. ISSN 0929-8673.

VOISIN, O. et al. ACUTE QT INTERVAL MODIFICATIONS DURING HYDROXYCHLOROQUINE-AZITHROMYCIN TREATMENT IN THE CONTEXT OF COVID-19 INFECTION. Mayo Clinic Proceedings, 2020, Elsevier.
WANG, C. et al. A novel coronavirus outbreak of global health concern. The Lancet, v. 395, n. 10223, p. 470-473, 2020. ISSN 0140-6736.

ZHENG, W.; SUN, W.; SIMEONOV, A. Drug repurposing screens and synergistic drug-combinations for infectious diseases. British journal of pharmacology, v. 175, n. 2, p. 181191, 2018. ISSN 1476-5381.

ZHENG, Y.-Y. et al. COVID-19 and the cardiovascular system. Nature Reviews Cardiology, v. 17, n. 5, p. 259-260, 2020. 